\title{
Tumor Diagnosis by Genetic Markers Protein P-53, p16, C-MYC, N- MYC, Protein K-Ras, and Gene Her-2 Neu is this possible? Tumor Diagnosis by Genetic Markers C-MYC, N-MYC, Protein P-53, p16
}

\author{
HANY A. AL-HASSANY'1, ALI H. ALBU-RGHAIF², MEENA A. NAJI ${ }^{3}$ \\ ${ }^{1}$ Ph.D. Student of Pharmacology, College of Medicine, University of Baghdad, Lecturer in Alnukhaba University, Baghdad, Iraq. \\ ${ }^{2}$ M.Sc. Medicinal Chemistry department of Pharmacy, Ashur University Collage, Baghdad, Iraq. corresponding author Hany akeel. \\ ${ }^{3}$ Doctor Diploma in Family Medicine, Department of Family Medicine, College of Medicine, University of Baghdad, Baghdad, Iraq. \\ Correspondence to: Hay Alqadisia, Baghdad, Iraq. Postcode: 1000., Email : hany_akeel2000@yahoo.com,Phone 009647733331443
}

\begin{abstract}
Currently, cancer is among the leading causes of death in the world. It is the correct diagnosis that gives the patient a better chance of healing. The detection of genetic markers, macromolecules that are produced directly by the tumor or by the host, has the potential to be one of the diagnostic tests. This paper is a review article, with the objective of addressing tumor markers c-MYC, n-MYC, protein p-53, protein Kras, and the gene HER-2 neu regarding the diagnosis and prognosis of patients with cancer, studies demonstrated that c-MYC is expressed in several malignant tumors because it affects proliferation and cellular metabolism, P-53 in breast and lung tumors is overexpressed, Regarding p16 it has been suggested that cytoplasmic also overexpression as kind of cancer predictor. K-ras is used to determine survival time and free time for neoplasm. It is an effective marker for monoclonal antibody therapy. The marker HER-2 neu and N-MYC are indicative of poor prognosis, conclusions: application of these markers is being increasingly used for expressing specificity and sensitivity in different areas of tumor characterization, in addition to being related to the therapeutic conduct to be followed in tumor treatment. Keywords: genetic marker; p16; Tumor marker; cancer diagnosis;p53
\end{abstract}

\section{INTRODUCTION}

Cancer is among the leading causes of death in the world, it is estimated that more than 12 million people are diagnosed with cancer every year (1).

Early diagnosis increases the chances of cure, survival, and quality of life, the markers, as well as other complementary tests of diagnosis, are of great importance, As an example of complementary tests, it has the initial anamnesis, according to (2), Radiographs, tomography's, Computed tomography, ultrasound, fibro bronchoscopy, and bone scintigraphy (3). The causes of cancer development are varied due to many factors; internal and external to the organism (4). That acts in carcinogenesis, enabling cells to become neoplastic. A neoplastic cell has metabolic advantages and altered biological capacities caused by the activation, mutation, or deletion of protooncogenes (5).

Proto-oncogenes are genes with the biological functions of proliferation, differentiation, and cell survival that make up the human genome and can mutate spontaneously, gene amplification, retrovirus action, or sequence translocation specific (6).

Mutations in proto-oncogenes generate the creation of oncogene, sequences related to carcinogenesis, which have the ability to produce oncoproteins, therefore; proteins are unable to exercise their natural functions because they are defective (7). Tumor markers are macromolecules that can help in the diagnosis of cancer since they are substances that can be produced directly by the tumor or by the body (8). They can be identified by biochemical means, immunohistochemistry, or genetic testing, for the search for oncogenes, genes tumor suppressors, and genetic alterations (9). The use of molecular techniques has helped to clarify the steps involved in carcinogenesis (10).
The p53 and K-ras markers, the proto-oncogene of the ras families, are often related to various cancers and environmental agents (11). Cancer marker HER-2 neu is an indicator of poor prognosis of carcinomas, more promising in relation to the possibility of choosing a treatment directed to the characteristics of the patient (12). The proto-oncogenes of the family myc, c-myc, N-myc and L-myc are also tumor markers. C-myc has the expression that is elevated in tumoral situations and $\mathrm{N}$-myc is related to a low response to treatment chemotherapy (13).

Studies report that the p53 protein and the HER-2 neu gene have been associated with the progression of tumors. (14) Marked p53 expression was observed in breast and lung tumors.

However, several studies demonstrated that many participants forming a heterogeneous group detailed that in cases with more p53 gene expression, there was a poor prognosis. However, other studies with more homogeneous groups did not obtain the same results (15), HER-2 neu also showed increased expression in cancer neoplasms breast. (16)

Overexpression of the HER-2 neu gene can be identified in $20 \%$ of cancers breast, (17) and in $33 \%$ of cases of lung carcinoma (18). However, according to studies, the expression of the HER-2 neu gene is linked to the accumulation of p53 protein, suggesting that the presence of these markers may indicate a poor prognosis for the disease. (19) Patients with HER-2 neu can be targeted for treatment with anti-HER 2 monoclonal antibodies (20).

Moreover, A poor prognosis was also analyzed in the association of p53, HER-2 neu, and K-ras, K-ras can be used to guide treatment mutation of the K-ras gene which can also influence the response for treatment. (21) Medicines such as anti-EGFR antibodies have the potential to be a successful treatment for the mutated gene (22). 
Since 2009, the American Society of Clinical Oncology has recommended that mAb treatment candidates be screened for K-ras mutations in codons 12 and 13. (23), because mutations in this gene identify patients who do not respond well to therapy with anti-EGFR drugs (24).

Mutated K-ras is seen in $15 \%$ to $20 \%$ of cancer cases, as in carcinoma pancreatic, colorectal, lung, and ovaries. In the case of lung cancer, it was found that patients with the mutation are less likely to survive (25). According to studies, the c-MYC oncogene has a significant role in malignant tumors, accounting for at least $40 \%$ of neoplasms such as bladder and cervical carcinoma, breast, ovarian, and prostate carcinoma, and lymphoma. (26)

Burkitt's disease and cervical adenocarcinoma, are being likely to appear up to $100 \%$ proto-oncogene c-MYC, whereas the others mentioned can vary from $5 \%$ to $96 \%$, Like c-MYC, n-MYC is also highly expressed in many types of cancers (27). Currently, neuroblastoma has n-MYC as it is the best marker that can be amplified by $50 \%$ in the most advanced cases. (28)

Furthermore, the detection of n-MYC in tumor diagnosis is associated with a poor patient prognosis and a poor response to chemotherapy treatment (29).

Cancers are complicated and differentiated processes, tumors in the same organ can have distinct molecular and genetic characteristics. Therefore; the identification of biomarkers can indicate the prognosis of the disease, which is associated with the survival rate, and appropriate therapeutic indication individually.

The present paper has the main objective which is to understand the tumor markers and genetic factors, their performance in determining neoplasia, and evaluating reliability in the clinical diagnosis and prognosis of tumors. The labels for the MYC gene family c- and n-MYC, the p53 marker protein, the K-ras protein, and the HER-2 neu gene were chosen for their representativeness in various neoplastic illness investigations.

\section{METHODOLOGY}

A bibliographic survey was carried out, using as keywords "Tumor markers" and "biomarkers" in google scholar, PubMed, dissertations, and thesis in the period from 1994 to 2021 in, English and Spanish. Thus, aiming to identify the markers of tumor cells c-MYC, n-MYC, protein $\mathrm{p}-53, \mathrm{p}$ 16 , protein K-ras, and the gene HER-2 neu as mechanisms of tumor diagnosis.

Tumor Markers: Tumor markers include circulating cellular products, encompassing: genetic material (DNA and RNA), serum proteins, enzymes, metabolites, proteins, carcinoembryonic, receptors, oncoproteins, proteins expressed by genes suppressors, even tumor cells can be investigated as markers $(32,33)$

These products are identified in patients with neoplasia. These indicators are produced by the body in reaction to disease or the tumor itself and can be found by research in the patient blood, urine, or even tissues. A tumor marker ideal should have characteristics such as only being clinically present in patients with malignant tumors, not being detected at high levels in patients with conditions as a malignant tumor, inflammatory or infectious conditions, and present high concentrations in the onset phase of the neoplasia so that the diagnosis was possible precocious. Proteolytic activity, proliferation speed, and degree of dispersion of determining the serum concentration of the measured tumor marker. For the tumor to be considered to be of clinical value, it must be specific, sensitive and it is clinical application can be found in association with neoplasms (30).

Tumor markers can be used in screening, clinical staging, tumor location, prognosis assessment, differential diagnosis, detection of relapses, and treatment follow-up. For the analysis of tumor markers it is important to take into account the half-life of the tumor marker, the metabolism, and excretion, the possible presence of ectopic markers and if possible perform the association of more than one marker so that sensitivity and specificity clinical diagnosis is more reliable (31).

P53 and P16 protein: The p53 gene is located on the short arm of chromosome 17 (17p). It is a gene of approximately $20 \mathrm{~Kb}$ which gives rise to a primary transcript ( $m R N A)$ of $2.8 \mathrm{~Kb}$. The un-mutated p53 gene is responsible for encoding a nuclear transcription factor. The gene transcribes the $53 \mathrm{kDa}$ phosphoprotein (32), which has the function of "Guardian of the genome" having fundamental importance in tumor suppression controlling cell proliferation, by regulating the cell cycle the cell growth in the G1-S interphase, which leads to apoptosis cells with DNA damage - this factor binds to p21 to induce its expression and inhibit the cell cycle to progress (33).

The oncogenic activity of p53 depends on the mutation in the reading sequence that promotes guaninethymine transversion (G-T), affecting p53 protein production and p21 activation capacity. That mutation is usually related to exposure to environmental carcinogens and can happen in different regions of the sequence. The oncogenic action of p53 is highly associated with solid tumors and their aggressiveness in humans according to parameters histological and biological and may be related to breast cancer (34) and lung cancer its mutant version has a longer half-life than the wild, which culminates in high levels of cells malignant organisms (35).

The clinical significance of assessing the level of $p 16$ expression in hyperplastic diseases of the endometrium and ductal carcinoma of the mammary gland has been established: in this case, it has been suggested that cytoplasmic overexpression of p16 is a kind of cancer predictor. In addition, the p16 protein is normally regarded as a marker of cell aging and is an inhibitor of cell division, which can occur in response to hyperproliferative signals, DNA damage, and oxidative stress (33).

According to Russell Vang et al., In addition to p53 and p16, the most important molecular markers for the diagnosis of vulvar cancer (50), apart from p53 and p16, is the WT1 marker, a protein product of the tumor growth springs of the same name, localized on chromosome 11 and consisting of 11 exons. WT1 is a transcription factor and is involved in the development of tissues derived from the mesoderm, in particular the urinary tract (51). WT1 is a protein that regulates the expression of a number of genes that are involved in cell proliferation and differentiation. Mutations of the corresponding gene, as well as high 
proliferative activity, can cause increased WT1 expression both in cancer tissue and in precancerous lesions. (52)

K-ras protein: The K-ras gene (Kirsten Rat Sarcoma Viral Oncogene Homologue) located in the arm short of chromosome 12, is part of the ras family (K-ras, $\mathrm{H}$-ras, $\mathrm{N}$ ras) of proteins of guanosine diphosphate/guanosine triphosphate (GDP / GTP) bond acting as a transducer of the signal. Indispensable component for regulating intracellular signaling pathways through a plurality of receptors, among them the EGFR (Epidermal Growth Factor Receptor). Guanine nucleotide exchange factors (GEFs) and guanosine triphosphatase activating proteins influence its activity. (36).

The ras protein, part of the G protein family, is the key to proliferation, differentiation, and apoptosis. They are activated by connection to GTP by GEFs, where their production is stimulated through EGFR growth factors. Inactivation occurs when ras GTP is hydrolyzed by intrinsic GTPase to ras-GDP (37). When active have greater compatibility with molecules that mostly activate signaling cascades.

Intracellular, among them, the rat sarcoma virus / Mitogen-activated protein pathway kinase (ras / MAPK) and the Phosphoinositide-3-kinase pathway (PI3K / AKT). In the normal physiological conditions, a constancy is maintained between the activity of GEFs and GAPs, important for the normal cellular mechanism of the tissue (38).

Mutations in the K-ras gene are usually somatic. The most frequently happens in exon 2 (codons 12 and 13), changing the guanine/adenine that during the formation of protein replaces the amino acid. Exon mutations may occur less often 3 (codons 61) and exon 4 (codons 146) (23).

Mutations can cause changes in protein conformation, overexpression of the EGFR factor, resulting in an everactive ras protein, leading to activation of signaling pathways with consequent abnormal cell growth, proliferation, and differentiation (39). Mutations can also change the relationship between the activity of GEFs and GAPs, generating a marked reduction in intrinsic GTPase activity, making it resistant to GAPs (40)

Oncogene c-MYC: It is classified as a proto-oncogene, and it plays a key role in the coordination of processes that lead to cell proliferation and differentiation in healthy cells. (27).

The c-MYC protein has two nuclear localization sequences (NLS) and structural domains that are determined as a transcription factor. Being that the first 143 amino acids of the $\mathrm{N}$-terminal portion assimilate the transactivation domain (TAD) (41). Which are closely linked to the biological activities performed by $\mathrm{c}-\mathrm{MYC}$ and have a high standard of conservation among family members "there is a link, said to be essential for c-MYC to perform its activities, is the MAX protein, which works as a transcriptional activator(42).

According to Zhao R et al. c-MYC is an oncogene of apoptotic capacity, and therefore; it has a short lifetime, around 30 minutes, which makes an effective gene regulator. (43)

Despite all its quality in preventing cancer, amplification of c-MYC it cannot be widely done in the clinic, because of neuroblastoma variables that do not have this amplification but have varying degrees of lethality (44).
In short, the c-MYC gene interferes in the cell cycle, and it can be by several means, in the $G 1$, in cell apoptosis, cell immortalization, angiogenesis, in energy metabolism, and cell adhesion, therefore; all these processes mentioned can interfere in the development of a neoplasm (45) and may suffer interference at these stages of the cell cycle to deplete a tumor. Inadequate activation of c-MYC can develop neoplasms, this activation happens by different means, being they chromosomal translocations, as seen in Burkitt's lymphoma; consequently, of its expression; stimulation of gene transcription, as observed in colon carcinomas; among others (45).

Oncogene n-MYC: According to Hald $\varnothing$, et.al the n-MYC gene is located in the distal part of the short arm of chromosome 2 (2p24), which may indicate rapid tumor progression, behavior aggressive, and a high risk of recurrence. This oncogene encodes a transcription factor that once in the nucleus, it generates a delay in cell differentiation promoting replication and apoptosis (44). Amplification of the n-MYC gene is associated with structural genetic disorders, related to frequent $1 p$ deletion in many neuroblastomas, and an increase of $17 p$ in about $80 \%$ of primary neuroblastomas (46).

HER-2 neu gene: The proto-oncogene called HER-2 neu can be found in the literature as c-erbB-2 and HER2. It comes from the human epidermal growth factor receptor due to its broad correlation with the growth factor receptor (RFC) (17). It's inserted on chromosome $17 q$ and performs the function of encoding the transmembrane glycoprotein that acts as a receptor of the RFC family and has specific tyrosine kinase activity (47). Its molecular weight is $185 \mathrm{Kd}$. (48)

There are several methods for determining overexposure of the HER-2 neu gene. (49) stated that methods such as "Western blot and immunoassays are used enzymes, or the amplification of HER-2 neu, such as Southern blot, slot blot, dot blot, in situ hybridization with polymerase chain reaction (PCR) and in situ hybridization by fluorescence (FISH) ".

\section{CONCLUSION}

The application of these markers for tumor diagnosis and prognosis is increasingly being used due to research and positive results. The markers described in this literature review, K-ras, p53, p-16, c-MYC, k nMYC, and HER-2 neu, expressed specificity and sensitivity in different areas of tumor characterization. However, there are many studies with controversial results. Therefore; it is important to have more specific studies and with appropriate methodologies for results reliable, consistent and accurate on tumor markers, given the relevance of these products. Even among many unanswered questions, events molecules that assist carcinogenesis have aided in the diagnosis and prognosis adequate and have shown importance to assist in the definition of the therapeutic lineage to be followed. Thus, tumor markers are an important ally in tumor detection in conjunction with other known diagnostic methods.

Acknowledgment: High gratitude to the collaboration and assistance of everyone in the department of pharmacology 
Faculty of the Medicine University of Baghdad for their support.

Disclaimer: None

Conflicts of interest: None

Source of Support: This research received no specific grant from any funding agency in the public, commercial or not-for-profit sectors

\section{REFERENCES}

1. Fitzmaurice C, Dicker D, Pain A, Hamavid H, Moradi-Lakeh M, Maclntyre MF, et al. The Global Burden of Cancer 2013. JAMA oncology. 2015;1(4), 505-527.).

2. Lacombe J, Mangé A, Bougnoux AC, Prassas I, Solassol J. A multiparametric serum marker panel as a complementary test to mammography for the diagnosis of node-negative earlystage breast cancer and DCIS in young women. Cancer Epidemiology and Prevention Biomarkers. 2014;23(9), 18341842).

3. Nath A, Neyaz Z, Hashim Z, Agrawal V, Richa M.. Role of Percutaneous Computed Tomography-guided Lung Biopsy in Non-resolving Consolidation and Identification of Clinical and High-resolution Computed Tomography Characteristics Predicting Outcome. Journal of clinical imaging science. 2019; (9).

4. Blackadar CB. Historical review of the causes of cancer. World journal of clinical oncology. 2016;7(1), 54.).

5. Aunan JR, Cho WC, Søreide K. The biology of aging and cancer: a brief overview of shared and divergent molecular hallmarks. Aging and disease,. 2017; 8(5), 628.).

6. Ala'a HM. Cytotoxic effect of Datura stramonium extract on cancer cell lines. . The Iraqi Journal of Veterinary Medicine. 2010; 34(1), 131-138.)

7. Alberti L, Carniti C, Miranda C, Roccato E, Pierotti MA. RET and NTRK1 proto-oncogenes in human diseases.. Journal of cellular physiology. 2003;195(2), 168-186.).

8. Wang N, Wang J, Zhao X, Chen $\mathrm{H}, \mathrm{Xu} \mathrm{H}$, Bai L, et al. Highly sensitive electrochemical immunosensor for the simultaneous detection of multiple tumor markers for signal amplification. Talanta. 2021;226, 122133.).

9. Huang RS, Haberberger J, Severson ED, uncan DL, Hemmerich A, Edgerly C, et al. A pan-cancer analysis of PD L1 immunohistochemistry and gene amplification, tumor mutation burden and microsatellite instability in 48,782 cases. Modern Pathology. 2021;34(2), 252-263.).

10. Varadhachary GR, Abbruzzese JL, Lenzi R. Diagnostic strategies for unknown primary cancer. Cancer: Interdisciplinary . Interdisciplinary International Journal of the American Cancer Society. 2004;100(9), 1776-1785.).

11. Roy H. AKT proto-oncogene overexpression is an early event during sporadic colon carcinogenesis. Carcinogenesis. 2002;23(1):201-205.

12. Sadasivan R, Morgan R, Jennings $S$, Austenfeld M, Stephens $\mathrm{R}$, Noble M. Overexpression of Her-2/neu may be an indicator of poor prognosis in prostate cancer.. The Journal of urology. 1993;150(1), 126-131.).

13. Nau M, Brooks B, Battey J, Sausville E, Gazdar A, Kirsch I et al. L-myc, a new myc-related gene amplified and expressed in human small cell lung cancer. Nature. 1985;318(6041):6973.

14. Hafidha RR, Husseinb SZ, MalAllahc MQ, Abdulamird SA, Abu Bakar F. A high-throughput quantitative expression analysis of cancer-related genes in human HepG2 cells in response to limonene, a potential anticancer agent. Curr Cancer Drug Targets. 2018;(18, 1-9).

15. Pastinen T, Sladek R, Gurd S, Sammak AA, Ge B, Lepage P, et al. A survey of genetic and epigenetic variation affecting human gene expression. . Physiological genomics. 2004;16(2), 184-193.).
16. Bacus SS, Ruby SG, Weinberg DS, Chin D, Ortiz R, Bacus JW. HER-2/neu oncogene expression and proliferation in breast cancers. . The American journal of pathology. 1990;137(1), 103.).

17. Ali SM, Carney WP, Esteva FJ, Fornier M, Harris L, Köstler WJ, et al. Serum HER-2/neu and relative resistance to trastuzumab-based therapy in patients with metastatic breast cancer. Cancer: Interdisciplinary Internati. Interdisciplinary International Journal of the American Cancer Society. 2008; 113(6), 1294-1301).

18. Molina R, Jo J, Filella X, Bruix J, Castells A, Hague M, et al. Serum levels of C-erbB-2 (HER-2/neu) in patients with malignant and non-malignant diseases.. Tumor biology. 1997; 18(3), 188-196.).

19. Rashed MM, Ragab NM, Galal MK. The Expression of p53, CD44, Ki-67, and HER-2/neu Markers in Gastric Cancer and Its Association with Histopathological Indicators: A Retrospective Study. Asian Pacific Journal of Cancer Prevention: APJCP. 2007; 21(6), 1607).

20. Vernieri $C$, Milano $M$, Brambilla $M$, Mennitto A, Maggi $C$ Cona MS, et al. Resistance mechanisms to anti-HER2 therapies in HER2-positive breast cancer: Current knowledge, new research directions and therapeutic perspectives. Critical reviews in oncology/hematology. 2019;(139, 53-66).

21. Bongiorno PF, Whyte RI, Lesser EJ, Moore JH, Orringer MBIBDG. Alterations of K-ras, p53, and erbB-2/neu in human lung adenocarcinomas. The Journal of thoracic and cardiovascular surgery. 1994;107(2), 590-595.).

22. Uchibori K, Inase N, Araki M, Kamada M, Sato S, Okuno Y, et al. Brigatinib combined with anti-EGFR antibody overcomes osimertinib resistance in EGFR-mutated non-small-cell lung cancer. . Nature communications. 2017;(8(1), 1-16.).

23. Zekri J, Al-Shehri A, Mahrous M, Al-Rehaily S, Darwish T, Bassi S, et al. Mutations in codons 12 and 13 of K-ras exon 2 in colorectal tumors of Saudi Arabian patients: frequency, clincopathological associations, and clinica. Genet Mol Res. 2017;16(10.4238)).

24. Hoshi H, Hiyama G, Ishikawa K, Inageda K, Fujimoto J, Wakamatsu $A$, et al. Construction of a novel cell-based assay for the evaluation of anti-EGFR drug efficacy against EGFR mutation. . Oncology reports. 2017; 37(1), 66-76.).

25. Yashiro M, Nishioka N, Hirakawa K. K-ras mutation influences macroscopic features of gastric carcinoma. . Journal of Surgical Research. 2005;124(1), 74-78.).

26. Hawksworth $D$, Ravindranath $L$, Chen $Y$, Furusato $B$, Sesterhenn IA, McLeod DG, et al. Overexpression of C-MYC oncogene in prostate cancer predicts biochemical recurrence. Prostate cancer and prostatic diseases. 2010;13(4), 311315.)

27. Elbadawy M, Usui T, Yamawaki H, Sasaki K. Emerging roles of C-Myc in cancer stem cell-related signaling and resistance to cancer chemotherapy: a potential therapeutic target against colorectal cancer. International journal of molecular sciences. 2019;20(9), 2340).

28. Schwab M. Amplification of $\mathrm{N}$-myc as a prognostic marker for patients with neuroblastoma. . In Seminars in cancer biology. 1993; No. 1( 4): p. 13-18.

29. Joshi VV, Larkin EW, Holbrook CT, Silverman JF, Norris HT, Cantor $\mathrm{AB}$, et al. Correlation between morphologic and other prognostic markers of neuroblastoma a study of histologic grade, DNA index, N-myc gene copy number, and lactic dehydrogenase in patients in the pediatric oncology group. Cancer. 1993; 71(10), 3173-3181).

30. Sargent D, Allegra C. Issues in clinical trial design for tumor marker studies.. In Seminars in oncology. 2002;( p. 222-230).

31. Duffy MJ. Clinical uses of tumor markers: a critical review. Critical reviews in clinical laboratory sciences. 2001; 38(3), 225-262).

32. Alkuraishy HM, Al-Gareeb Al, Al-hussaniy HA. Doxorubicininduced cardiotoxicity: molecular mechanism and protection 
by conventional drugs and natural products. Int $\mathrm{J}$ Clin Oncol Cancer Res. 2017; 2(2), 31-44.).

33. Sigal A, Rotter V. Oncogenic mutations of the p53 tumor suppressor: the demons of the guardian of the genome. . Cancer research,. 2000; 60(24), 6788-6793.).

34. AL KURAISHY HM, AL GAREEB ALII, NAJI HA. FEBUXOSTAT MODULATES OXIDATIVE AND APOPTOTIC PATHWAYS IN ACUTE DOXORUBICIN INDUCED CARDIOTOXICITY: AN EXPERIMENTAL ANIMAL MODEL STUDY.. Asian J Pharm Clin Res,. 2019;12(4), 73-76).

35. Finlay CA, Hinds PW, Tan TH, Eliyahu D, Oren M, Levine AJ. Activating mutations for transformation by p53 produce a gene product that forms an hsc70-p53 complex with an altered half-life. Molecular and cellular biology. 1988;8(2), 531-539).

36. Jinesh GG, Sambandam V, Vijayaraghavan S, Balaji K, Mukherjee S. Molecular genetics and cellular events of KRas-driven tumorigenesis. ,. Oncogene. 2018;37(7), 839846.).

37. Hall BE, Bar-Sagi D, Nassar N. The structural basis for the transition from Ras-GTP to Ras-GDP. . Proceedings of the National Academy of Sciences,. 2002;99(19), 12138-12142.).

38. Lu H, Guo Y, Gupta G, Tian X. Mitogen-activated protein kinase (MAPK): new insights in breast cancer. Journal of Environmental Pathology, Toxicology and Oncology. 2019;(38(1).).

39. Dempke WC, Heinemann V. Ras mutational status is a biomarker for resistance to EGFR inhibitors in colorectal carcinoma. . Anticancer research. 2010;30(11), 4673-4677.).

40. Haigis KM. KRAS alleles: the devil is in the detail. . Trends in cancer. 2017;3(10), 686-697.).

41. Gregory MA, Hann SR. C-Myc proteolysis by the ubiquitinproteasome pathway: stabilization of c-Myc in Burkitt's lymphoma cells.. Molecular and cellular biology. 2000;20(7), 2423-2435.).
42. Zheng K, Cubero F, Nevzorova Y. C-MYC-Making Liver Sick: Role of c-MYC in Hepatic Cell Function, Homeostasis and Disease. Genes. 2017;8(4):123.

43. Zhao R, Liu Y, Wu C, Li M, Wei Y, Niu W, et al. BRD7 promotes cell proliferation and tumor growth through stabilization of c-Myc in colorectal cancer. . Frontiers in Cell and Developmental Biology. 2021;9, 955.).

44. Hald Ø, Olsen L, Gallo-Oller G, Elfman L, Løkke C, Kogner P et al. Inhibitors of ribosome biogenesis repress the growth of MYCN-amplified neuroblastoma. Oncogene. 2018;38(15):2800-2813.

45. Jeong YJ, Hoe HS, Cho HJ, Park KK, Kim DD, Kim CH, et al. Suppression of c-Myc enhances p21WAF1/CIP1-mediated G1 cell cycle arrest through the modulation of ERK phosphorylation by ascochlorin.. Journal of cellular biochemistry. 2018;119(2), 2036-2047).

46. Cao $Y$, Jin $Y, Y u$ J, Wang J, Yan J, Zhao Q. Research progress of neuroblastoma related gene variations. . Oncotarget. 2017;8(11), 18444.).

47. Ceglie G, Del Baldo G, Agolini E, Rinelli M, Cacchione A, Del Bufalo F, et al. Cancer Predisposition Syndromes Associated With Pediatric High-Grade Gliomas. Frontiers in pediatrics. 2020;(8).

48. Ghassami E,VJ,MM,NM,\&HSM. Biodistribution, Safety and Organ Toxicity of Docetaxel-Loaded in HER-2 Aptamer Conjugated Ecoflex® Nanoparticles in a Mouse Xenograft Model of Ovarian Cancer.. Recent patents on nanotechnology. 2019;13(1), 49-58).

49. Wang K, Guan C, Yu J, Jin X, Sun L, Zheng L, et al. Prognostic value of HER-2/neu expression in epithelial ovarian cancer: a systematic review and meta-analysis. Oncotarget. 2017;8(43), 75528.). 